Société d'histoire de la révolution de 1848 et des

révolutions du XIXe siècle

$37 \mid 2008$

L'ère victorienne revisitée

\title{
Erik GRIMMER-SOLEM, The Rise of Historical Economics and Social Reform in Germany, 1864-1894
}

\author{
Marie-Bénédicte Vincent
}

\section{OpenEdition}

\section{Journals}

Édition électronique

URL : http://journals.openedition.org/rh19/3552

DOI : $10.4000 /$ rh 19.3552

ISSN : $1777-5329$

Éditeur

La Société de 1848

\section{Édition imprimée}

Date de publication : 15 novembre 2008

Pagination : 185-242

ISSN : 1265-1354

Référence électronique

Marie-Bénédicte Vincent, «Erik GRIMMER-SOLEM, The Rise of Historical Economics and Social Reform in Germany, 1864-1894 », Revue d'histoire du XIXe siècle [En ligne], 37 | 2008, mis en ligne le 01 décembre 2008, consulté le 22 septembre 2020. URL : http://journals.openedition.org/rh19/3552 ; DOI : https:// doi.org/10.4000/rh19.3552

Ce document a été généré automatiquement le 22 septembre 2020.

Tous droits réservés 


\title{
Erik GRIMMER-SOLEM, The Rise of Historical Economics and Social Reform in Germany, 1864-1894
}

\author{
Marie-Bénédicte Vincent
}

\section{RÉFÉRENCE}

Erik GRIMMER-SOLEM,The Rise of Historical Economics and Social Reform in Germany, 1864-1894, Oxford, Clarendon Press, Oxford Historical Monographs, 2003, 338 p. ISBN : 978-0-199-26041-6. 75 livres sterling.

1 On disposait déjà du livre de David Lindenfeld sur l'institutionnalisation des sciences politiques au XIX ${ }^{e}$ siècle en Allemagne ${ }^{1}$. Le présent ouvrage le complète, en explorant la dimension sociale de "l'école historique » (historische Schule), ce courant économique qui prône la réforme sociale dans les années 1860-1890 où se fonde l'Empire bismarckien.

Dans cette période de grands changements (seconde industrialisation, expansion démographique, urbanisation), la théorie économique classique connaît en effet un discrédit qui rend possible l'émergence de paradigmes alternatifs. Parmi eux, l'école historique se fonde sur une démarche statistique et empirique qui privilégie l'étude historique des structures de la vie économique. Abordant les sujets brûlants du moment (le protectionnisme, l'intervention de l'État ou la diffusion du socialisme), elle popularise au sein des couches bourgeoises l'idée d'une régulation sociale progressive de l'économie, pour aboutir à une société égalitaire de couches moyennes, la fameuse Mittelstandsgesellschaft (sans classes). Cette éthique sociale s'inscrit plus largement dans la tentative de la bourgeoisie de trouver une réponse légale et institutionnelle à la crise de la société urbaine et industrielle.

3 Le livre veut donc explorer le lien méthodologique et pratique entre cette école et les projets de réforme sociale des milieux progressistes. Il remet en cause l'image d'une 
discipline économique divisée à la fin du XIX ${ }^{\mathrm{e}}$ siècle entre les partisans du mouvement ouvrier et ceux de la société bourgeoise. En effet, les professeurs engagés de l'école historique, ces "socialistes de la chaire» (Kathedersozialisten) comme les appelait ironiquement la presse, étaient loin de former un groupe homogène. Derrière Gustav Friedrich Schmoller (1838-1917) leur chef de file, Lujo Brentano (1844-1931), Adolf Held (1844-1880) et Georg Friedrich Knapp (1842-1926) - les figures clé auxquelles s'attache le livre - n'avaient ni les mêmes sujets d'investigation, ni les mêmes orientations politiques. Leurs relations étaient lâches et leur localisation pas exclusivement berlinoise. L'auteur cherche donc ce qui a pu les réunir.

Pour autant, l'approche d'Erik Grimmer-Solem ne relève pas de l'histoire des idées. Au contraire, il veut faire une histoire sociale de ce courant intellectuel, en analysant les trajectoires des individus, leurs positions dans le monde scientifique et social et leurs liens associatifs et éditoriaux, afin de comprendre leurs postures méthodologiques, scientifiques et politiques. Son objectif est d'analyser le processus social de la production de connaissances.

5 Les sciences économiques ont émergé en Allemagne à partir des sciences camérales (discipline administrative comportant un enseignement pratique dans la gestion des domaines, du commerce et des mines). L'évolution vers l'économie politique (Nationalökonomie), conduisit quelques universités telles Munich ou Tübingen à fonder des facultés distinctes de sciences politiques (Staatswissenschaften). Mais en règle générale, l'économie fut intégrée aux facultés de philosophie, où elle bénéficia d'une grande autonomie. La pluralité du système universitaire rendit possible la coexistence de plusieurs variantes de Staatswissenschaften, d'autant qu'à la différence du droit, les gouvernements n'avaient pas fixé d'examens d'État pour certifier un niveau de compétences. C'est le doctorat qui servait de label, mais, là encore, ses exigences variaient d'une université à l'autre. La grande flexibilité de la discipline explique la liberté de son développement : diffusion des statistiques, introduction de la forme du séminaire et élargissement de l'éventail de cours vers la question sociale et ouvrière.

Gustav Friedrich Schmoller fut ainsi le premier à mettre en place à Halle et Strasbourg des séminaires de statistiques impliquant une dimension de recherche. Il était très influencé par le célèbre séminaire de Ernst Engel (1821-1896) à Berlin, au sein du Bureau prussien des statistiques. Engel, député libéral au Parlement prussien, avait innové dans cette institution devenue indépendante en 1862 en proposant dans son enseignement des matériaux empiriques et des excursions. Il fut cependant contraint à la démission par Bismarck en 1882, malgré sa réputation internationale. C'est dans ce séminaire que furent formés Held, Knapp et Brentano.

7 L'autre institution clef est l'Association de politique sociale (Verein für Sozialpolitik). Créée en 1873 par Schmoller, Brentano, Held, Knapp et d'autres, elle est devenue l'organisation de réforme sociale la plus importante d'Allemagne. Elle coordonnait des recherches empiriques (sa collection, les Schriften des Vereins für Sozialpolitik publie 140 volumes jusqu'en 1914) et organisait des congrès annuels à Eisenach jusqu'en 1876, puis dans d'autres villes, favorisant le dialogue entre professeurs d'économie, statisticiens et administrateurs. Le Verein représentait donc une sorte de privatisation de l'enquête sociale menée auparavant par les ministères ou les bureaux statistiques des États. Il travailla pour le Reichstag en amont de l'élaboration des lois. Deux autres associations ont joué un rôle important dans la structuration de l'école historique : la Staatswissenschaftliche Gesellschaft (fondée par Schmoller en 1883 pour encourager la 
discussion entre économistes et hauts fonctionnaires) et la Gesellschaft für staatswissenschaftliche Fortbildung, société de formation continue pour fonctionnaires fondée en 1902 également par Schmoller et Althoff. Enfin, il faut mentionner le rôle stratégique des éditeurs, les deux principaux pour l'école économique historique étant Carl Geibel (1842-1910), propriétaire de Duncker et Humblot à Berlin/Leipzig et Gustav Fischer (1845-1910), député libéral de Prusse et fondateur des éditions Fischer à léna.

$8 \mathrm{Au}$ total, le livre montre bien comment l'école historique a contribué à la professionnalisation des économistes en Allemagne, par une émancipation de la discipline à l'Université et par la mise en place de revues et sociétés savantes. Après la Première guerre mondiale, l'école historique perdit de son attractivité à l'étranger du fait de l'isolement de l'Allemagne au plan scientifique. Elle fut redécouverte dans les années 1960-1970 par ceux qui s'interrogeaient sur l'influence des statistiques et de l'économie historique sur le développement des sciences sociales dans les pays anglosaxons.

\section{NOTES}

1. . David Lindenfeld, The Practical Imagination. The German Science of State in the Nineteenth-Century, Chicago, The University of Chicago Press, 1997. 